\title{
Impacts of Perinatal Induced Photothrombotic Stroke on Sensorimotor Performance in Adult Rats
}

\author{
T. BRIMA ${ }^{1}$, A. MIKULECKÁ ${ }^{1}$, J. OTÁHAL $^{1}$ \\ ${ }^{1}$ Department of Developmental Epileptology, Institute of Physiology, Academy of Sciences \\ of the Czech Republic, Prague, Czech Republic
}

Received August 9, 2012

Accepted September 24, 2012

On-line November 22, 2012

\begin{abstract}
Summary
Perinatal ischemic stroke is a leading cerebrovascular disorder occurring in infants around the time of birth associated with long term comorbidities including motor, cognitive and behavioral deficits. We sought to determine the impact of perinatal induced stroke on locomotion, behavior and motor function in rats. A photothrombotic model of ischemic stroke was used in rat at postnatal day 7. Presently, we induced two lesions of different extents, to assess the consequences of stroke on motor function, locomotion and possible correlations to morphological changes. Behavioral tests sensitive to sensorimotor changes were used; locomotion expressed as distance moved in the open field was monitored and histological changes were also assessed. Outcomes depicted two kinds of lesions of different shapes and sizes, relative to laser illumination. Motor performance of rats submitted to stroke was poor when compared to controls; a difference in motor performance was also noted between rats with small and large lesions. Correlations were observed between: motor performance and exposition time; volume ratio and exposition time; and in the rotarod between motor performance and volume ratio. Outcomes demonstrate that photothrombotic cerebral ischemic stroke induced in early postnatal period and tested in adulthood, indeed influenced functional performance governed by the affected brain regions.
\end{abstract}

\section{Key words}

Photothrombosis - Perinatal ischemic stroke - Sensorimotor performance $\bullet$ Immature rats

\section{Corresponding author}

J. Otáhal, Institute of Physiology, Academy of Sciences of the Czech Republic, Vídeňská 1083, 14220 Prague 4, Czech Republic. Fax: +420-241062488.

E-mail: jotahal@epilepsy.biomed.cas.cz

\section{Introduction}

Perinatal ischemic stroke is the leading cerebrovascular disorder occurring in infants around the time of birth with pathological evidence of focal arterial infarction (de Vries et al. 1997, Nelson and Lynch 2004). Symptomatic perinatal ischemic stroke is experienced by about one in every 4000 term births (Estan and Hope 1997, Lynch and Nelson 2001) and is associated with high medical and social costs, due to long-term comorbidity (Sran and Baumann 1988, Sreenan et al. 2000). Despite a lack of symptoms during early postnatal periods, studies has shown that the numbers of reported neonatal stroke cases are on the increase in some areas (Edwards and Azzopardi 2000, Miller et al. 2004, Wu et al. 2005) thus the actual incidence is likely higher than the above written value. Up to $27 \%$ of infants with these brain injuries later in life develop numerous consequent pathologies as well as behavioral and functional deficits. Among these are speech delays, hemi-paresis and hemisensory impairments (Westmacott et al. 2009, Vinay et al. 2005, Hattori et al. 2000). Clinically, little is known concerning long-term behavioral outcome after neonatal stroke (Westmacott et al. 2009), this fact holds true also in experimental studies. There is a shortage of stroke models focused purely on long-term neurobehavioral and functional outcomes induced at an immature age and assessed in adulthood. Existing work of this nature documented a correlation between the degree of brain injury in neonatal rats and functional deficits such as asymmetries of limb placing, foot-faults and abnormality in the postural reflex tests (Bona et al. 1997). Although neonatal stroke is increasingly being studied, most of the 
foundation of our understanding on functional and behavioral endpoints comes from research done in adult animal models. In order to study perinatal stroke, several methods to induce ischemic brain damage have been elaborated in immature rats at postnatal day (P) 7 (Ashwal et al. 1995, Renolleau et al. 1998, Rice et al. 1981). Research evinced that rat's brain at this age is as mature as the brain of a human fetus in the third trimester; with regard to parameters such as number of synapses, neurochemical development and cortical organization (Hagberg et al. 1997); making it the appropriate age for this study. Hypoxic ischemic (HI) based methods are dominating in this field, but prove to be technically challenging to perform at an early age resulting in inconsistent infarct volume and high mortality rates. In contrast, photothrombosis is a less invasive method that can be performed at an immature age (Maxwell and Dyck 2005). This method is based on a thrombus-producing photochemical reaction of photosensitive dyes activated by green laser light that typically produces a consistent infarct of a specific location and size (Watson and Prado 2009, Karhunen et al. 2007, Matějovská et al. 2008).

In the present study we used the Bengal Rose model of photothrombotic stroke to induce two ischemic lesions of different extends, at postnatal day (P) 7. This was done in order to assess consequences of perinatal stroke on motor performance, behavioral and functional responses, such as posture and motor coordination in adulthood. To this end, animals were submitted to four simple behavioral tests (rotarod, bar holding, inclined grid, and ladder rung walking) sensitive to sensorimotor changes caused by various brain injuries. Moreover, locomotor activity expressed as distance moved in the open field (OF) was monitored.

\section{Material and Methods}

\section{Animals}

Experiments were performed in male albino Wistar rats $(n=139)$, provided by the Institute of Physiology of the Academy of Sciences of Czech Republic. At postnatal day (P) 7 the animals were brought to the experimental room and divided into four groups: animals $(\mathrm{n}=30)$ exposed to laser light for $5 \mathrm{~min}$ (BR_5 min); animals $(\mathrm{n}=38)$ exposed to laser light for $30 \mathrm{~s}$ (BR_30 s); sham-operated controls (Sham) treated with saline $(n=31)$ and intact control group $(C, n=40)$. Behavioral tests were performed at $\mathrm{P}$ 67. A group of seventy-eight rats $\left(B R \_5 \mathrm{~min}=20, \mathrm{BR} \_30 \mathrm{~s}=19\right.$, Sham $=19$ and $\mathrm{C}=20$ ) were submitted to rotarod, bar holding and inclined grid tests. A second group of sixty-one rats (BR_5 $\min =10$, BR_30 $\mathrm{s}=19$, Sham $=12$ and $\mathrm{C}=20$ ), were submitted to ladder rung walking tests and open field (OF). A subset of twenty-one animals, $n=7$ from each experimental group, were used to confirm the ischemic injury. Rats were housed in standard plastic cages in a temperature-controlled environment of $22 \pm 1{ }^{\circ} \mathrm{C}$, humidity $50-60 \%$ with a 12 -h light/dark cycle (lights on at 6 a.m.) with free access to food and water. All efforts were made to minimize animal suffering and to reduce the number of animals used.

The protocol of the experiments was approved by the animal Care and Use Committee of the Institute of Physiology, Academy of Sciences of the Czech Republic (v.v.i.) to be in agreement with the Animal Protection Law of the Czech Republic, which is fully compatible with the guidelines of the European Community Council directives 86/609/EEC.

\section{Induction of photothrombosis}

Cortical brain lesions were induced by photothrombosis according to Watson and Prado (2009) with slight modifications. Before the procedures started rats where weighted and marked. They were anesthetized with isoflurane (1.5-5\%). A midline scalp incision was made and the scalp was retracted laterally to expose the skull. Freshly prepared Bengal Rose solution (Sigma ${ }^{\circledR}, \mathrm{BR}$ $10 \mathrm{mg} / \mathrm{ml}, 0.15 \mathrm{ml}$ per animal) was injected into the exposed right jugular vein, rats were then transferred within $1 \mathrm{~min}$ to a stereotaxic frame and their brains were exposed (through an intact skull) to Green laser light (Roithner Laser, Austria $50 \mathrm{~mW}$ output) for $60 \mathrm{~s}$ at $0.5 \mathrm{~s}$ on/off cycles. The center of the light beam was focused on the left sensorimotor cortex, $1.8 \mathrm{~mm}$ from Bregma anterior posteriorly (AP) and $2.8 \mathrm{~mm}$ laterally (L) extending to the midline medially $\mathrm{AP}=1.8 \mathrm{~mm}, \mathrm{~L}=2.8 \mathrm{~mm}$ (Paxinos and Watson 1998). The incisions were immediately sutured and collodium was applied on the suture. Animals were allowed to recover from anesthesia for about $45 \mathrm{~min}$ before returning to their mother. Sham-operated controls followed the same protocol, except that saline was used instead of Bengal Rose injection before laser light illumination. Two animals were removed from experiment due to procedural problems during surgery. Body temperatures were monitored and maintained at $37^{\circ} \mathrm{C}$ throughout the surgery. The health conditions of animals were checked regularly. 


\section{Behavioral testing}

Behavioral testing was performed in adult animals, 60 days after photothrombosis. The deficits of weight supported stepping, paws coordination, endurance, muscle strength, skilled walking, were assessed with a battery of simple tests adopted from our previous work (Mikulecká and Mareš 2002, Tichá et al. 2011). Before behavioral testing, all animals were weighted and left to adapt to the experimental room for one hour.

\section{Rotarod test}

Motor coordination and equilibrium was observed using an automatic rotarod treadmill unit, set to rotate at a constant $5 \mathrm{rpms}$ and placed $30 \mathrm{~cm}$ above a landing platform. The unit consisted of a horizontal rod $(10 \mathrm{~cm}$ in diameter $11 \mathrm{~cm}$ long) covered with thick plaster to increase its roughness. The rats were placed individually on the rotating rod with their heads directed against the direction of rotation. Three trials were performed in a close succession. Maximal score in maintaining equilibrium was arbitrarily fixed at $60 \mathrm{~s}$. The mean of three trials was taken as a score. A video camera was placed in front of the rotarod, to observe the behavioral strategies used by an animal to maintain equilibrium. Two strategies were observed - passive rotation and walking. The passive rotation consisted in hanging on the rotarod and being passively rotated. During the walking behavior the rat walked asynchronously (the movement of the paws was not perfectly coordinated) and synchronously (the rat walked evenly, the paws being perfectly coordinated).

\section{Bar holding}

An animal was held by the nape and its forepaws were allowed to touch a wooden bar $(25 \mathrm{~cm}$ long, $1 \mathrm{~cm}$ in diameter, suspended $25 \mathrm{~cm}$ above a padded soft surface). Time of fore- and hind-limb grasping was recorded with a limit of $60 \mathrm{~s}$. The mean of the three grasping latencies was taken as the score.

\section{The inclined grid}

Turning on the inclined grid was assessed using a modification of the procedure described by Marshall (1982). A grid (75 cm with x $100 \mathrm{~cm}$ height) made of stainless was held in a horizontal position. A rat was placed on it, approximately in the center. Then, the grid was lowered until it attained a negative inclination of $30^{\circ}$ with respect to the horizontal plane. The latency to turn on the grid to $180^{\circ}$ was measured for a maximum of $60 \mathrm{~s}$. If the rat failed to turn on the grid, the latency was set to $60 \mathrm{~s}$. The mean of three turning latencies was taken as a score.

\section{The ladder rung walking}

The ladder rung walking test is used to assess motor and locomotor performances specifically skilled walking, forelimb and hind limb placing, stepping, and inter-limb co-ordination and balance (Metz and Whishaw 2002). In the present study we used this test to assess the ability of rats to successfully cross the runway. A horizontal ladder walking apparatus that consisted of side walls made of clear Plexiglas and metal rungs $(3 \mathrm{~mm}$ diameter), that could be inserted to create a floor with a minimum distance of $1 \mathrm{~cm}$ between rungs was used. The ladder was elevated $30 \mathrm{~cm}$ above the ground with a empty starting cage and a refuge (home cage) at the end. The width of the alley was adjusted to the size of the animal, to prevent the animal from turning around. The time to cross the runway in one trial with regular gaps and another trial with irregular gaps was assessed. The rats were tested with a limit of $60 \mathrm{~s}$.

\section{Open field}

The open field (OF) test was performed in a square arena $(45 \times 45 \times 30 \mathrm{~cm})$, with a camera installed above the center. Immediately after a rat was placed in the center of the arena; locomotor behavior was recorded automatically by a computerized system (Etho Vision Noldus Information Technology) for $5 \mathrm{~min}$. Locomotor activity expressed as distance moved (cm) was calculated.

To reduce any lingering olfactory cues all devices were cleaned after each rat was tested.

\section{Histology}

One week after behavioral testing, animals were overdosed with urethane (2 g/kg i.p.), and perfused transcardially with ice cold $0.01 \mathrm{M}$ phosphate-buffered saline (PBS; pH 7.5) followed by $4 \%$ formaldehyde. The brains were removed and post fixed in $4 \%$ formaldehyde at $4{ }^{\circ} \mathrm{C}$ and then sequentially soaked in $10 \%, 20 \%$ and $30 \%$ sucrose. The brains were then frozen in dry ice and stored at $-70^{\circ} \mathrm{C}$. Coronal sections $(50 \mu \mathrm{m}$ thick; Cryocut Leika 1600) were cut through the entire brain and every fifth section was collected and placed in $4 \%$ formaldehyde. To assess cell morphology, brain sections were derived from all investigated rats, mounted onto 
gelatin-coated slides and stained with cresyl violet or Nissl staining (Otáhal et al. 2005). Slides were coverslipped and assessed using a light microscope (Olympus ${ }^{\circledR}$ AX 70 microscope with bright field optics).

\section{Lesion evaluation}

To evaluate the magnitude of lesions; ratios between hemispheres (affected/contralateral) were calculated. Volumes of these hemispheres were stereologically estimated by Cavalieri principle and point-grid probe in the Ellipse software (ViDiTo, Slovakia). Briefly, every fifth slice $(50 \mu \mathrm{m}$ thick) was obtained from a cutting series of slices throughout the brain. These slices were stained with Nissl and placed under a stereomicroscope, to capture their images, with a digital camera. A set of slices from one brain and a measuring scale bar were captured using the same magnification and camera setting. The obtained images included both hemispheres; this allowed us to use the same settings in the point-grid for both hemispheres, affected and non-affected contralateral hemispheres. Spacing of the point-grid probe was set to $0.2 \mathrm{~mm}$.

\section{Statistics}

Statistical analysis was performed with (Sigma Stat3.5®SPSS) program. To assess the difference in tissue volume, non parametrical Mann-Whitney test was used. Pearson Product Moment Correlation was used to detect correlations between motor performance and volume ratio and between volume ratio and duration of laser light exposure. Sensorimotor performance data were analyzed using one-way ANOVA with post hoc StudentNewman-Keuls Method. The differences in the ratio of correct response (animals crossed the ladder rung walking within the $60 \mathrm{~s}$ ) were evaluated by means of $\mathrm{Chi}^{2}$-test. All data was expressed as mean \pm SEM and statistical significance $\mathrm{P}<0.05$ was accepted.

\section{Results}

\section{Morphology of the photothrombotic lesions}

Two different exposition times to green laser light ( $30 \mathrm{~s}$ and $5 \mathrm{~min}$ ) focused on sensorimotor cortex, responsible for sensorimotor limb function (CastroAlamancos and Borrel 1995), were chosen to induce focal cerebral ischemia in 7 days old rats. The shorter exposition time (30 s-BR_30 s) induced lesions which maturates to diameters ranging from $0.3-2.5 \mathrm{~mm}$ when assessed in adult animals, penetrating through all cortical

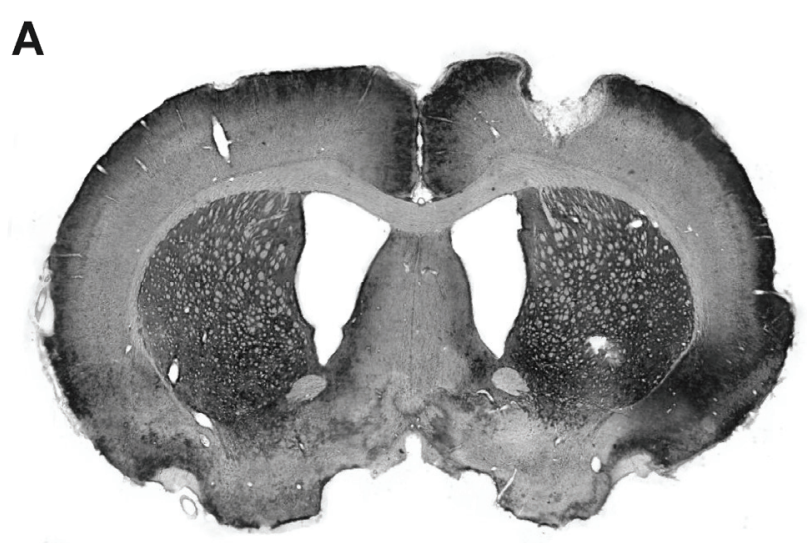

B

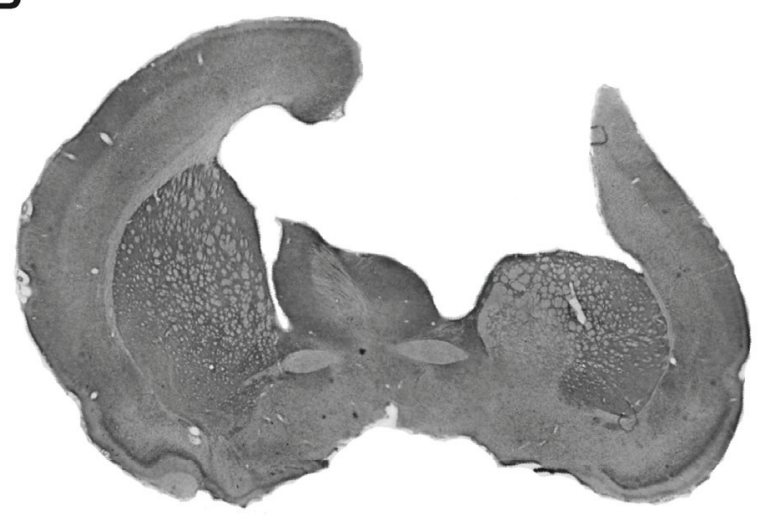

C

Ratio of the volume of hemispheres

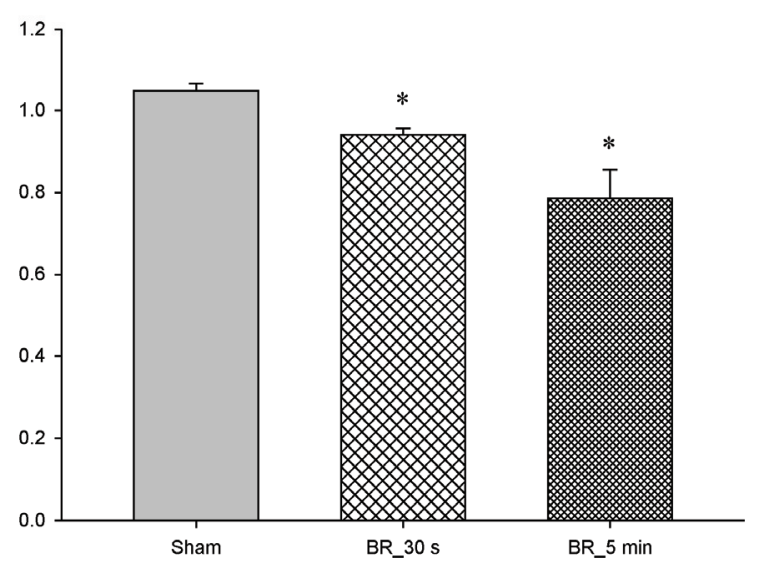

Fig. 1. Representative, Nissl-stained coronal brain section of photothrombotic infarct induced in $\mathrm{P7}$ and assessed in adult rats showing, the involvement of all cortical layers in BR_30 s (A) and BR_5 min (B) including the underling subcortical white matter. Sections obtained at $50 \mu \mathrm{m}$ intervals throughout the lesion were used to calculate volume ratio (C) of hemispheres of shamoperated controls, BR_30 s and BR_5 min.

layers leaving subcortical white matter intact (Fig. 1A). The longer exposition time (5 min - BR_5 min) induced larger lesions of $2.4-3.7 \mathrm{~mm}$ in diameter involving all cortical layers including the underlying white matter and 

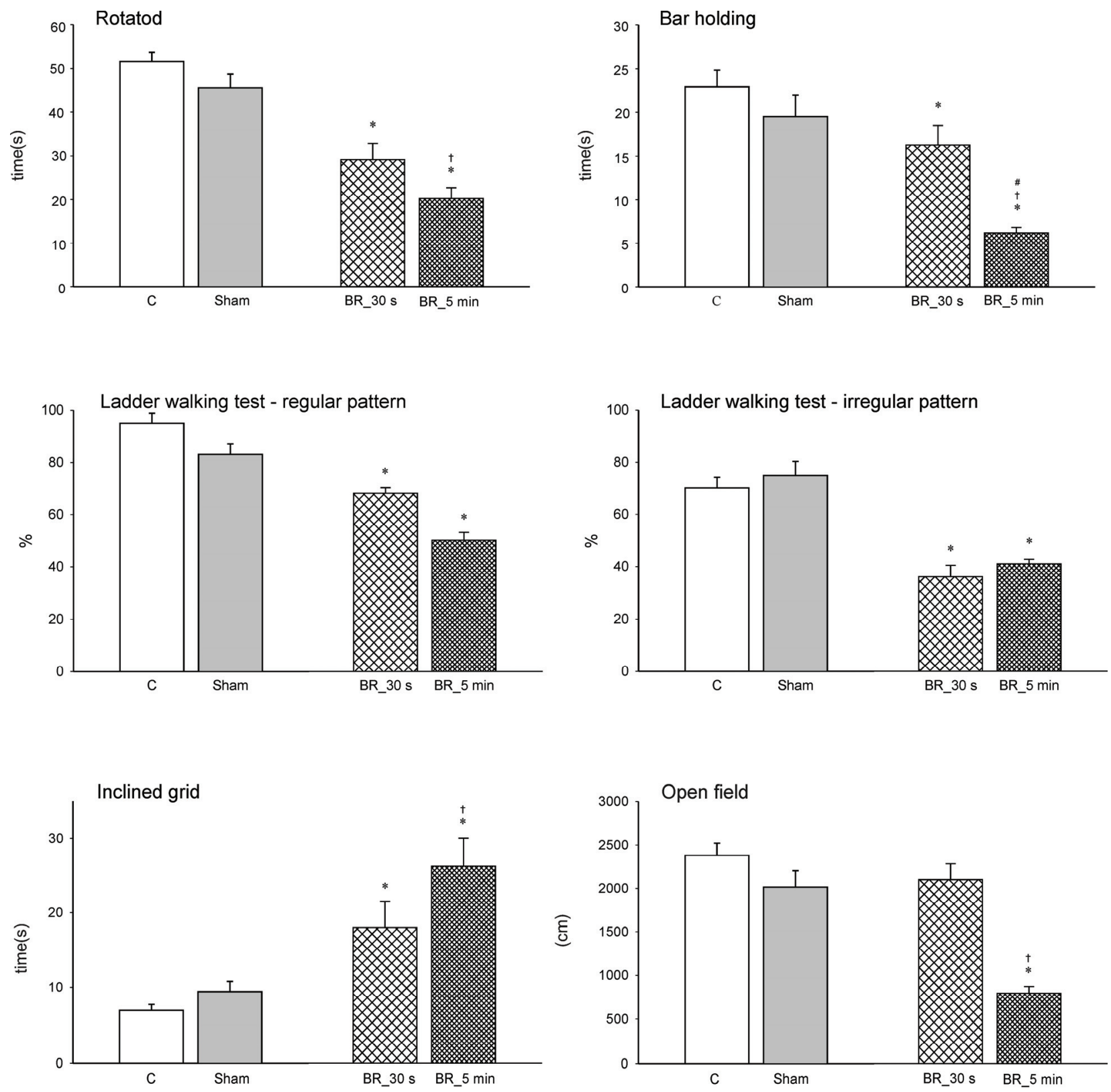

Fig. 2. Effects of photothrombosis induced at $P 7$ on the sensorimotor behavior of adult rats. Abscissa: $C$ (controls); Sham (sham operated controls); BR_30 s (group exposed to laser light for $30 \mathrm{~s}$ ); and BR_5 min (group exposed to laser light for 5 min). Ordinate: mean + S.E.M., * Compared to both C and Sham, \# Compared to Sham, † Compared to BR_30 s.

subcortical structures (e.g. striatum) of affected hemisphere (Fig. 1B). Volume ratios between hemispheres (affected/contralateral) were calculated; the animals with large lesion presented a mean volume ratio of $(0.79 \pm 0.07)$ ischemic hemisphere versus the contralateral hemisphere, while those with small lesion had a mean volume ratio of $(0.94 \pm 0.02)$. Mann-Whitney test revealed a significant difference in volume ratio between rats with longer exposition time and controls, intact (C) and sham-operated (Sham); also between both controls (C and Sham) and rats with shorter exposition time (Fig. 1C). Pearson Product Moment Correlation also revealed significant negative correlations between volume ratio and exposition time. All control animals, intact and sham-operated, presented no structural evidence of cerebral damage.

\section{Behavioral testing}

The results of behavioral testing are summarized in Figure 2.

\section{Rotarod test}

The ability to coordinate and balance on a rotating drum was tested in adult rats, 60 days after 
photothrombosis. The overall analysis of the time spent on the rotarod revealed significant main effects of experimental condition $(F(3,74)=24.62, p<0.001)$. Both groups of rats with either small lesions or large lesions walked for a significantly shorter time on the rotarod (29.16 $\pm 3.79 \mathrm{~s}$ and $20.23 \pm 2.5 \mathrm{~s}$, respectively) compared to controls (C) $51.57 \pm 2.22 \mathrm{~s}$ and Sham-operated rats $45.6 \pm 3.09 \mathrm{~s}$. There was significant positive correlation between the time spent on the rotarod and volume ratio. The animals with large lesions and longer exposition time spent less time on the rotarod compared to animals with small lesions and exposed to laser light for shorter time.

\section{Bar holding}

Strength and grip of both fore- and hind-limbs was assessed in this test. A significant main effect of photothrombotic lesion on the time spent on the bar was demonstrated $(F(3,74)=10.79, p<0.001)$. The time spent holding the bar was significantly shorter in both groups of rats subjected to stroke; rats with shorter exposition time $(16.3 \pm 2.2 \mathrm{~s})$ and rats with longer exposition time $(6.2 \pm 0.65 \mathrm{~s}) \quad$ compared to both sham-operated $(19.5 \pm 2.5 \mathrm{~s})$ and intact controls $(22.95 \pm 1.89 \mathrm{~s})$.

\section{Inclined grid}

Typically, geostatic abilities, immobility, catalepsy and the latencies to turn to $180^{\circ}$ as function of time spend on a grid that is incrementally raised to increasing angles is assessed. At present the latency of rats to turn $180^{\circ}$ on an inclined grid was recorded for a maximum of $60 \mathrm{~s}$. These latencies significantly increased in both groups subjected to stroke $(F(3,74)=10.78$, $p<0.001)$, with values of $(29.16 \pm 3.79 \mathrm{~s})$ in rats with longer exposition times and $(20.23 \pm 2.5 \mathrm{~s})$ in those with shorter exposition times as compared to both intact $(9.4 \pm 1.4 \mathrm{~s})$ and $(6.9 \pm 0.8 \mathrm{~s})$ sham-operated controls. The animals with longer exposition time had an increased latency to turning on the grid compared to the animals exposed for shorter time to laser light.

\section{Ladder rung walking}

The time to complete ladder rung walking task was assessed in a trial with regular gap and another trial with irregular gap arrangement. In this test, $95 \%$ of intact controls, $83 \%$ of sham-operated rats, $68 \%$ of BR_30 s and $50 \%$ of BR_ 5 min animals crossed the runway with regular gap pattern within $60 \mathrm{~s}\left(\mathrm{Chi}^{2}=9.4, p=0.02\right)$. In the trial using irregular gap pattern, $70 \%$ controls, $75 \%$ sham-operated animals, $37 \%$ BR_30 s and $41 \%$ BR_5 min group completed the task within $60 \mathrm{~s}\left(\mathrm{Chi}^{2}=10.3\right.$, $p=0.06)$. The ANOVA showed that the latency to cross the ladder with regular gaps was affected $(\mathrm{F}(3,57)=3.67$, $p=0.017$ ). Surprisingly, there were no statistically significant differences in the latency to cross the ladder between tested groups in both trials with regular or irregular gaps $(\mathrm{F}(3,57)=3.67, p=0.017 ; F(3,57)=1.74$, $p=0.017$ ) respectively.

\section{Open field}

Locomotor and exploratory behavior in rats placed in an open field arena was monitored for five minutes per animal. The distance moved in the OF revealed significant main effect of lesion size on locomotion in the animals subjected to stroke $(F(3,57)=13.69, p<0.001)$. The post-hoc test showed that only the animals with longer exposition time walked a shorter distance $(791.4 \pm 77.5 \mathrm{~cm})$ compared to all other groups; no significant differences were found between the animals with the small lesions $(2097.8 \pm 182.1 \mathrm{~cm})$ and both control groups (sham-operated 2015.0 $\pm 185.3 \mathrm{~cm}$ and intact controls $2378.0 \pm 144.4 \mathrm{~cm}$ ).

\section{Discussion}

The effect of cerebral ischemia has been analyzed by many authors, mostly assessing acute and chronic outcomes of lesions induced in adult animals, but rarely the impact of stroke induced in immature animals, and assessed in adulthood. Several reports showed that the immature brain reacts differently to ischemia than does the mature brain, for a review see (Aden et al. 2002, Saucier et al. 2007, Matsumori et al. 2006). Therefore, it is critical to use experimental models of an appropriate age (Aden et al. 2002). Lindner et al. (2003) and Bona et al. (1997) stressed the importance of long-term functional outcomes, to improve the validity of animal models of stroke rather than relying exclusively on acute consequences.

The present study investigated sensorimotor performances and locomotor activity of adult animals subjected to focal photothrombotic stroke at an early (P7) age. We adopted a technique developed initially for use in neonatal mice (Maxwell and Dyck 2005) to induce photothrombotic stroke in immature rats. Firstly, we focused on assessing whether early postnatal exposure to short and long laser light exposition time induces photothrombotic stroke of two different infract sizes in adulthood, and secondly, how they are related to 
sensorimotor performances in adulthood.

To date, the most frequently used neonatal stroke model of long-term neurobehavioral and functional outcomes is hypoxia-ischemia (HI) and middle cerebral artery occlusion (MCA-O) (Almli et al. 2000, Bona et al. 1997, Arteni et al. 2003, Balduini et al. 2000, Balduini et al. 2003, Northington 2006, Renolleau et al. 1998). These models were reported to have high mortality rates (Maxwell and Dyck 2005) and affect both animal's body weight and behavior (van der Staay 1998). For instance in a study by (Renolleau et al. 1998) all 7-day-old pups subjected to left MCA electrocoagulation with transient carotid occlusion lasting for more than 1 hour died during procedure; if occlusion lasted an hour of less, only $10 \%$ of pups died during the first 2 hours. In the present study, all animals survived until adulthood except two animals that died during surgery. Moreover, no differences in animal body weight that might affect motor performances were observed between the controls and animals subjected to stroke (data not shown).

Data from this study depicted two kinds of lesions; different in shape and size relative to laser light illumination. Exposure to this light for $30 \mathrm{~s}$ resulted in small lesion restricted to the sensorimotor cortex leaving the subcortical structures intact. While exposure for $5 \mathrm{~min}$ resulted in large lesions that involved all cortical layers including the underlying white matter and striatum of affected hemisphere. The large ischemic lesions had significantly less tissue remaining relative to the group with the small lesions. Sensorimotor ability (motor coordination), includes all processes that affects the brain's ability to synchronize the function of interrelated muscles. Synchronization of muscle movement occurs primarily in the cerebellum. The cerebral cortex is important in controlling the forelimbs and less important in control of the predominantly ambulatory hind-limb. Rats with destroyed cerebellum or inferior olivary complex are unable to perform complex sensorimotor tasks (Rondi-Reig et al. 1997). In this study, motor coordination was measured as a function of the total time each rat was able to remain on a rotating cylinder or the time rats were able to turn to $180^{\circ}$ on an inclined platform as the angle of inclination was progressively increased. Our results revealed that sensorimotor performance of rats post stroke was clearly affected when compared to controls. In the bar holding test, one of the most demanding tests for motor coordination and strength, impairments of motor performance were more expressed. Specifically, the animals with larger lesions were only able to grasp the bar for a very short time.

In the ladder rank walking test, arranged with a pattern of regular gaps, revealed no statistical differences in percentage to complete the task within $60 \mathrm{~s}$ among the tested groups. Similar results were also found in the task with irregular pattern. However, the percentage of animals among these groups that successfully completed the given task was decreased to a large extent. These results might be due to motor deficits coupled with decreased motivation to rejoin their cage mates.

In the open field test, animals with larger lesion walked a shorter distance compared to the other groups, suggesting a marked inhibition of locomotion. Surprisingly, among our animals, no signs of ataxia or loss of balance was observed. However, there was a clear relationship between sensorimotor impairments and lesion extent. These outcomes are contrary to previous observations that show no relationship between OF behavior and lesion severity (Chou et al. 2001). On the other hand, our results are in agreement with previous findings indicating that the animals exposed to laser for a longer time had the largest lesions and demonstrated the greatest sensorimotor and locomotion impairments (Sulejczak et al. 2007, Wood et al. 1996). In a combined analysis of controls and HI-subjected rats, a strong correlation between the degree of brain damage and longterm sensorimotor deficits was demonstrated (Bona et al. 1997). In their study, neurological functions were evaluated in a set of four sensorimotor tests performed between five and six weeks after hypoxia-ischemia; asymmetries of limb placing, foot-faults and abnormality in the postural reflex tests were detected. Other studies noted particular differences between behavioral outcomes and lesion size; Jones et al. (2008) observed large unilateral lesions in $\mathrm{HI}$ rats accompanied by reduction in forelimb grasping strength, increased number of foot faults in grid-walking test and forelimb asymmetry in cylinder test compared with sham-operated controls. On the other hand, Shanina et al. (2006) demonstrated that small lesions with intact subcortical layers resulted in significant sensorimotor impairments detectable only during the first two weeks after photothrombotic ischemic stroke. Similarly, Biernaskie et al. (2005) demonstrated that the degree of behavioral recovery in animals treated with lidocaine after endotelin-1 induced stroke was dependent on the infract size. In their study, animals with large lesions were unable to retrieve food pellets; whereas animals with small lesions returned to pre-ischemia performance levels. Our results generated from a 
photothrombotic model are in accordance with the data described above.

In conclusion, we demonstrated that photothrombotic cerebral ischemic stroke induced in the early postnatal period and tested in adult rats, indeed influenced sensorimotor performance governed by the affected brain region. This model makes it possible to mimic lesion variability, size and location as observed in most human stroke; providing an opportunity for longterm evaluation of behavioral and neuropathological endpoints. Future considerations may therefore be aimed at exploring and refining basic experimental issues; it will be of great interest to extend this study to analyze kinematic changes and task performance such as limb placing, foot faults and postural motor function in this particular model of stroke.

\section{Conflict of Interest}

There is no conflict of interest.

\section{Acknowledgements}

This study was supported by grant from Czech Science Foundation no. P303/10/0999 and project RVO67985823.

\section{References}

ADEN U, DAHLBERG V, FREDHOLM BB, LAI LJ, CHEN Z, BJELKE B: MRI evaluation and functional assessment of brain injury after hypoxic ischemia in neonatal mice. Stroke 33: 1405-1410, 2002.

ALMLI CR, LEVY TJ, HAN BH, SHAH AR, GIDDAY JM, HOLTZMAN DM: BDNF protects against spatial memory deficits following neonatal hypoxia-ischemia. Exp Neurol 166: 99-114, 2000.

ARTENI NS, SALGUEIRO J, TORRES I, ACHAVAL M, NETTO CA: Neonatal cerebral hypoxia-ischemia causes lateralized memory impairments in the adult rat. Brain Res 973: 171-178, 2003.

ASHWAL S, COLE DJ, OSBORNE S, OSBORNE TN, PEARCE WJ: L-NAME reduces infarct volume in a filament model of transient middle cerebral artery occlusion in the rat pup. Pediatr Res 38: 652-656, 1995.

BALDUINI W, DE ANGELIS V, MAZZONI E, CIMINO M: Long-lasting behavioral alterations following a hypoxic/ischemic brain injury in neonatal rats. Brain Res 859: 318-325, 2000.

BALDUINI W, MAZZONI E, CARLONI S, DE SIMONI MG, PEREGO C, SIRONI L, CIMINO M: Prophylactic but not delayed administration of simvastatin protects against long-lasting cognitive and morphological consequences of neonatal hypoxic-ischemic brain injury, reduces interleukin-1beta and tumor necrosis factoralpha mRNA induction, and does not affect endothelial nitric oxide synthase expression. Stroke 34: 2007-2012, 2003.

BIERNASKIE J, SZYMANSKA A, WINDLE V, CORBETT D: Bi-hemispheric contribution to functional motor recovery of the affected forelimb following focal ischemic brain injury in rats. Eur J Neurosci 21: 989-999, 2005.

BONA E, JOHANSSON BB, HAGBERG H: Sensorimotor function and neuropathology five to six weeks after hypoxia-ischemia in seven-day-old rats. Pediatr Res 42: 678-683, 1997.

CASTRO-ALAMANCOS MA, BORREL J: Functional recovery of forelimb response capacity after forelimb primary motor cortex damage in the rat is due to the reorganization of adjacent areas of cortex. Neuroscience 68: 793$805,1995$.

CHOU IC, TRAKHT T, SIGNORI C, SMITH J, FELT BT, VAZQUEZ DM, BARKS JD: Behavioral/environmental intervention improves learning after cerebral hypoxia-ischemia in rats. Stroke 32: 2192-2197, 2001.

DE VRIES LS, GROENENDAAL F, EKEN P, VAN HAASTERT IC, RADEMAKER KJ, MEINERS LC: Infarcts in the vascular distribution of the middle cerebral artery in preterm and fullterm infants. Neuropediatrics 28: 8896, 1997.

EDWARDS AD, AZZOPARDI DV: Perinatal hypoxia-ischemia and brain injury. Pediatr Res 47: 431-432, 2000.

ESTAN J, HOPE P: Unilateral neonatal cerebral infarction in full term infants. Arch Dis Child Fetal Neonatal Ed 76: F88-F93, 1997.

HAGBERG H, BONA E, GILLAND E, PUKA-SUNDVALL M: Hypoxia-ischaemia model in the 7-day-old rat: possibilities and shortcomings. Acta Paediatr Suppl 422: 85-88, 1997. 
HATTORI K, LEE H, HURN PD, CRAIN BJ, TRAYSTMAN RJ, DEVRIES AC: Cognitive deficits after focal cerebral ischemia in mice. Stroke 31: 1939-1944, 2000.

JONES NM, KARDASHYAN L, CALLAWAY JK, LEE EM, BEART PM: Long-term functional and protective actions of preconditioning with hypoxia, cobalt chloride, and desferrioxamine against hypoxic-ischemic injury in neonatal rats, Pediatr Res 63: 620-624, 2008.

KARHUNEN H, BEZVENYUK Z, NISSINEN J, SIVENIUS J, JOLKKONEN J, PITKANEN A: Epileptogenesis after cortical photothrombotic brain lesion in rats. Neuroscience 148: 314-324, 2007.

LINDNER MD, GRIBKOFF VK, DONLAN NA, JONES TA: Long-lasting functional disabilities in middle-aged rats with small cerebral infarcts, J Neurosci 23: 10913-10922, 2003.

LYNCH JK, NELSON KB: Epidemiology of perinatal stroke. Curr Opin Pediatr 13: 499-505, 2001.

MARSHALL JF: Sensorimotor disturbances in the aging rodent. J Gerontol 37: 548-554, 1982.

MATĚJOVSKÁ I, BERNÁŠKOVÁ K, KRÝSL D, MAREŠ J: Influence of melatonin pretreatment and preconditioning by hypobaric hypoxia on the development of cortical photothrombotic ischemic lesion. Physiol Res 57: 283$288,2008$.

MATSUMORI Y, NORTHINGTON FJ, HONG SM, KAYAMA T, SHELDON RA, VEXLER ZS, FERRIERO DM, WEINSTEIN PR, LIU J: Reduction of caspase-8 and -9 cleavage is associated with increased c-FLIP and increased binding of Apaf-1 and Hsp70 after neonatal hypoxic/ischemic injury in mice overexpressing Hsp70. Stroke 37: 507-512, 2006.

MAXWELL KA, DYCK RH: Induction of reproducible focal ischemic lesions in neonatal mice by photothrombosis. Dev Neurosci 27: 121-126, 2005.

METZ GA, WHISHAW IQ: Cortical and subcortical lesions impair skilled walking in the ladder rung walking test: a new task to evaluate fore- and hindlimb stepping, placing, and co-ordination. J Neurosci Methods 115: 169$179,2002$.

MIKULECKÁ A, MAREŠ P: NMDA receptor antagonists impair motor performance in immature rats. Psychopharmacology (Berl) 162: 364-372, 2002.

MILLER SP, MCQUILLEN PS, MCMAHAN TD, HAMRICK SE, BARKOVICH AJ, PARTRIDGE S, FERRIERO DM, AZAKIE A, KARL T: Timing of brain injury in newborns with congenital heart disease. Ann Neurol (Suppl 8) 56: S90, 2004.

NELSON KB, LYNCH JK: Stroke in newborn infants. Lancet Neurol 3: 150-158, 2004.

NORTHINGTON FJ: Brief update on animal models of hypoxic-ischemic encephalopathy and neonatal stroke. ILAR $J$ 47: 32-38, 2006.

OTÁHAL J, SUCHOMELOVÁ L, DRUGA R, KUBOVÁ H: Changes in cytochrome oxidase in the piriform cortex after status epilepticus in adult rats. Epilepsia 46 (Suppl 5): 89-93, 2005.

PAXINOS G, WATSON C: The Rat Brain in Stereotaxic Coordinates, fourth ed. Academic Press, San Diego, California, 1998.

RENOLLEAU S, GGOUN-ZOUAOUI D, BEN-ARI Y, CHARRIAUT-MARLANGUE C: A model of transient unilateral focal ischemia with reperfusion in the $\mathrm{P7}$ neonatal rat: morphological changes indicative of apoptosis. Stroke 29: 1454-1460, 1998.

RICE JE, VANNUCCI RC, BRIERLEY JB: The influence of immaturity on hypoxic-ischemic brain damage in the rat. Ann Neurol 9: 131-141, 1981.

RONDI-REIG L, HAYE-BOUCHAUD N, MARIANI J, CASTON J: Role of the inferior olivary complex in motor skills and motor learning in the adult rat. Neuroscience 77: 955-963, 1997.

SAUCIER DM, YAGER JY, ARMSTRONG EA, KELLER A, SHULTZ S: Enriched environment and the effect of age on ischemic brain damage. Brain Res 1170: 31-38, 2007.

SHANINA EV, SCHALLERT T, WITTE OW, REDECKER C: Behavioral recovery from unilateral photothrombotic infarcts of the forelimb sensorimotor cortex in rats: role of the contralateral cortex. Neuroscience 139: 1495$1506,2006$.

SRAN SK, BAUMANN RJ: Outcome of neonatal strokes. Am J Dis Child 142: 1086-1088, 1988.

SREENAN C, BHARGAVA R, ROBERTSON CM: Cerebral infarction in the term newborn: clinical presentation and long-term outcome. J Pediatr 137: 351-355, 2000. 
SULEJCZAK D, ZIEMLINSKA E, CZARKOWSKA-BAUCH J, NOSECKA E, STRZALKOWSKI R, SKUP M: Focal photothrombotic lesion of the rat motor cortex increases BDNF levels in motor-sensory cortical areas not accompanied by recovery of forelimb motor skills. J Neurotrauma 24: 1362-1377, 2007.

TICHÁ K, MIKULECKÁ A, MAREŠ P: Behavioral consequences of the mGlu5 receptor antagonist MTEP in immature rats. Pharmacol Biochem Behav 99: 619-625, 2011.

VAN DER STAAY FJ: Behavioral effects of stroke induced by occlusion of the middle cerebral artery (MCA) in rodents. In: The Study of Behavioral Dysfunctions: An Evaluation of Selected Animal Models. VAN DER STAAY FJ (ed), Graduate School of Behavioral and Cognitive Neurosciences (BCN), Groningen, The Netherlands, 2000, pp 145-209.

VINAY L, BEN-MABROUK F, BROCARD F, CLARAC F, JEAN-XAVIER C, PEARLSTEIN E, PFLIEGER JF: Perinatal development of the motor systems involved in postural control. Neural Plast 12: 131-139, 2005.

WATSON BD, PRADO R: Photochemically based models of focal experimental thrombotic stroke in rodents. In: Manual of Stroke Models in Rats. WANG-FISCHER Y (ed), CRC Press, Boca Raton, 2009, pp. 139-165.

WATSON BD, DIETRICH WD, BUSTO R, WACHTEL MS, GINSBERG MD: Induction of reproducible brain infarction by photochemically initiated thrombosis. Ann Neurol 17: 497-504, 1985.

WESTMACOTT R, MACGREGOR D, ASKALAN R, DEVEBER G: Late emergence of cognitive deficits after unilateral neonatal stroke. Stroke 40: 2012-2019, 2009.

WOOD NI, SOPESEN BV, ROBERTS JC, PAMBAKIAN P, ROTHAUL AL, HUNTER AJ, HAMILTON TC: Motor dysfunction in a photothrombotic focal ischaemia model. Behav Brain Res 78: 113-120, 1996.

WU YW, LYNCH JK, NELSON KB: Perinatal arterial stroke: understanding mechanisms and outcomes. Semin Neurol 25: 424-434. 2005. 